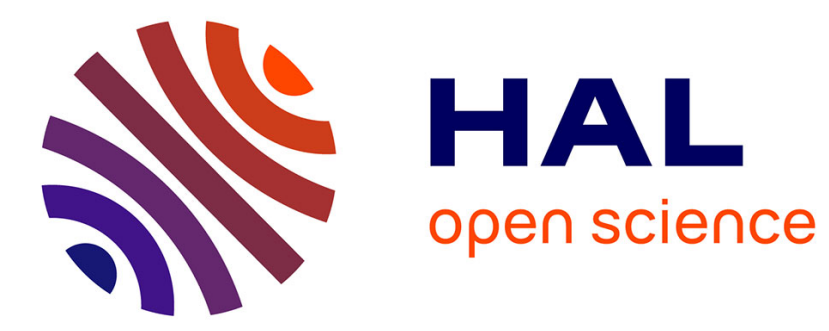

\title{
Automatic 3D face recognition using topological techniques
}

\author{
Chafik Samir, Jean-Philippe Vandeborre, Mohamed Daoudi
}

\section{To cite this version:}

Chafik Samir, Jean-Philippe Vandeborre, Mohamed Daoudi. Automatic 3D face recognition using topological techniques. IEEE International Conference on Multimedia \& Expo (ICME 2005), Jul 2005, Amsterdam, Netherlands. hal-00725593

\section{HAL Id: hal-00725593 \\ https://hal.science/hal-00725593}

Submitted on 27 Aug 2012

HAL is a multi-disciplinary open access archive for the deposit and dissemination of scientific research documents, whether they are published or not. The documents may come from teaching and research institutions in France or abroad, or from public or private research centers.
L'archive ouverte pluridisciplinaire HAL, est destinée au dépôt et à la diffusion de documents scientifiques de niveau recherche, publiés ou non, émanant des établissements d'enseignement et de recherche français ou étrangers, des laboratoires publics ou privés. 


\section{AUTOMATIC 3D FACE RECOGNITION USING TOPOLOGICAL TECHNIQUES}

\section{Chafik Samir, Jean-Phillipe Vandeborre}

\author{
MIIRE (INT / LIFL UMR CNRS 8022) \\ ENIC Télécom Lille1 \\ cité scientifique, rue G.Marconi \\ 59655 Villeneuve d'Ascq, France \\ \{chafik, vandeborre\}@enic.fr
}

\author{
Mohamed Daoudi \\ Université François-Rabelais \\ Laboratoire d'Informatique de Tours (EA 2101) \\ 64 avenue Jean Portalis \\ 37200 Tours, France \\ mohamed.daoudieuniv-tours.fr
}

\begin{abstract}
In this paper, we use the three-dimensional topological shape information for human face identification. We propose a new method to represent 3D faces as a topological graph. Fine registration of surfaces is done by first automatically finding topological connnected components, and then constructing its topological graph representing the important topological changes on the face. The similarity calculation between 3D faces is processed using coarse-to-fine strategy while preserving the consistency of the graph structures, which result in establishing a correspondance between the parts of faces. The experiments made with a 144 3D faces dataset show the efficiency of our approach.
\end{abstract}

\section{INTRODUCTION}

Automatic recognition of human faces from 2D intensity images has been studied extensively in the computer vision community. Facial variations due to illumination conditions, pose changes and different facial expressions make the recognition problem very challenging. Due to recent advances in both 3D acquisition systems and computational power of computers, it has become worthwhile to examine the advantages of 3D facial information in recognition [1].

Gordon [2] begins with a segmentation based on mean and Gaussian curvature. The nose region and ridge and valley lines from the segmentation are used to register the image to a standard pose.

Moreno et al. [3] extract features from regions and lines of interest, and apply classification using feature vector matching according to the minimum Euclidean distance classifier.

Srivastava et al. [4] presented an efficient technique to compare certain aspects of facial shapes using ideas from image analysis: a search for optimal subpace based on stochastic gradient algorithm on a Grassman manifold.

In [5] a minimum of three corresponding points is needed in order to calculate the rigid transformation between two sets of 3D points. The shape index at point $\mathrm{p}$ is calculated using the maximum $\left(k_{1}\right)$ and minimum $\left(k_{2}\right)$ local curvatures. A robust similarity metric is defined for matching, based on an Iterative Closest Point (ICP) registration process. Results are given for matching a database of $183 \mathrm{D}$ face models.

Lee and Milios [6] segment convex regions in the range image based on the sign of the mean and Gaussian curvatures, and create an Extended Gaussian Image (EGI) for each convex region. A matching between a region in a probe image (image to be recognized) and in a gallery image (image enrolled in the system) is done by correlating EGIs. A graph matching algorithm incorporating relational constraints is used to establish an overall match of probe image to gallery image. Convex regions are believed to change shape less than other regions in response to changes in facial expression. This gives this approach some ability to cope with changes in facial expression.

Blanz et al. [7] present a system that combines deformable 3D models with a computer graphics simulation of projection and illumination, given a single image of person, their algorithm estimates $3 \mathrm{D}$ shape, texture, and relevant scene parameters.

In this paper, we propose a system that combines geometrical information and topological properties, first the most important changes on the mesh are localized on topological connected components, secondly the mean curvature is calculated on each component. A topological augmented graph is constructed by defining topological neighboring relation between two nodes, where each node represents a connected component, according to a Morse function, and its mean curvature is added as an attribute. To compare two 3D faces the similarity of their MRGs is determined by an enriched matching by the topological configurations and the geometrical attributes. The paper is organized in the following way. In section 2, the problem of global and partial similarity is exposed. In sections 3 and 4, our approach based on Reeb graph is detailed. And finally, the experiments and results 
are presented.

\section{GLOBAL AND PARTIAL SIMILARITY}

In order to handle global and local properties of a 3D face simultaneously, the selected search key must include a concise representation of the 3D face, must catch features of the shape well, and must be computed automatically and robustly for a general 3D face database.

In order to satify such conditions, we propose to use the topological Reeb graph of a 3D face as a search key.

\section{REEB GRAPH}

Graph is widely adopted in areas such as matching [8], mesh representation [9], computer animation [10] and other applications.

However, the requirements for a graph differ with applications. For example, objects matching may need the graph to preserve principal features such as geometric information and topological properties, so we can regard these graphs as major keys and use them to find face similarities.

A traditional Reeb graph definition [11] is the object representation with a graph such that its nodes are connected components according to a Morse function. This function can be choosen [10] for different goals: object retrieval [9], terrain application [8]. Figure 1 shows a classical example of a Reeb graph obtained on a 3D bitorus according to a height function as the Morse function. Please note that figure 1(b) shows the connected components within the meaning of the selected Morse function.
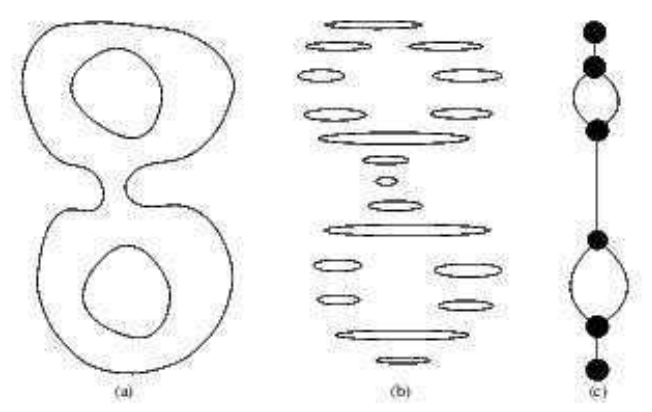

Figure 1. (a) a bitorus (b) connected components (c) Reeb graph

\subsection{Theoretical background}

According to the Morse theory, a continuous function defined on a closed surface characterizes the topology of the surface on its critical points [12]. Therefore, a Reeb graph can be obtained assuming a function $f$ calculated over the $3 \mathrm{D}$ face surface. The multiresolutional aspect results from the dichotomic discretization of the function values and from the hierarchical collection of Reeb graphs defined at each resolution.

The Reeb graph of figure 1(c) is a skeletal graph encoding compact manifolds in 3D space. It operates on the surface of a 3D face and gives a 1D representation for the face. In mathematical terms, a Reeb graph is defined as follows:

Let $f$ be real-valued function on a compact manifold $M$. The Reeb graph of $f$ is the quotient space on the graph of $f$ in $M$ by the equivalence relation space defined by:

$$
x_{1} \equiv x_{2} \Longleftrightarrow\left(\mathrm{f}\left(x_{1}\right)=\mathrm{f}\left(x_{2}\right)\right)
$$

and $x_{1}$ and $x_{2}$ are in the same connected component of $f^{-1}\left(f\left(x_{1}\right)\right)$.

A node in a Reeb graph represents a level set curve on a manifold defined by a real function $f$. When $f$ has no degenerated critical points on the manifold, it is called Morse function [13]. Figure 3 illustrates some examples of Reeb graph for a 3D face surface. To give an intuitive explanation, the Morse function $f$ here is the height function which simply returns the height value of a point on the subject surface. From bottom to top, the level set curves on the surface expand, split, merge and become smaller, resulting in the graph depicted in figure 1(c). The Reeb Graph gives a compact description of the evolution of level set curves and reveals the topological changes implied in the evolution.

\subsection{Multiresolution Reeb Graph (MRG)}

The Reeb graph is obtained by partitioning the 3D surface following regular intervals of $f$ values, and linking the connected regions to each other. In each interval, a node is associated to a set of connected triangles (cf. figure 1).

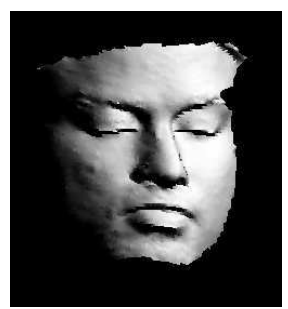

(a)

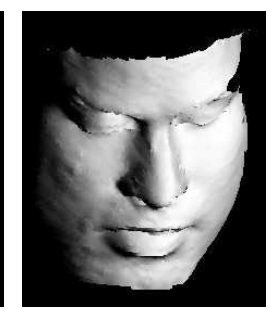

(b)

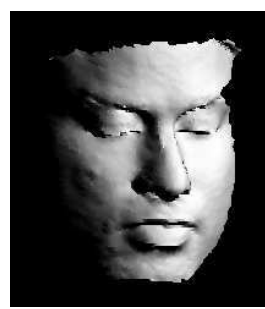

(c)
Figure 2. Three deformed faces from a same class

The number $N$ of connected components obtained on $3 \mathrm{D}$ face surface is chosen according to the level $l$ of multiresolution with the relation $N=2^{l}$. Figure 3 shows two MRGs in level 1 and level 3 calculated on the same subject fig. 2(b). If the level is high, the corresponding MRG is finer and record detailled information of the 3D face, but the recognition becomes hard to improve, in this work we found that level 5 give accurate results. 


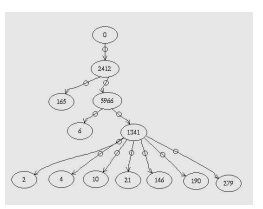

level 1 of fig.2(c)

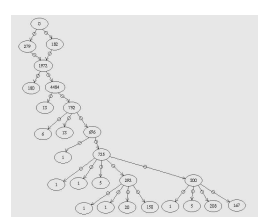

level 3 of fig.2(b)

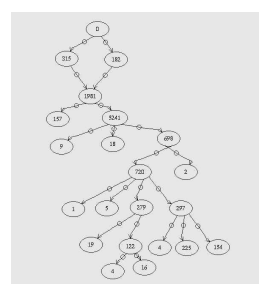

level 3 of fig.2(c)
Figure 3. Corresponding MRG to figure 2

\subsection{Size invariance}

Some existing approaches to 3D face recognition involves sensitivity to size variation [1]. We present in this paper an algorithm to construct the topological graph where the nodes are the connected components with a multiresolutional level, calculated as proportion to $(\min , \max )$ values of the function. The choice of level determines the number of the connected components on the mesh. The interval of values start with min value of $Z$ axis and stop in max value, Face with different sizes will have an equal number of connected componants. In figures 3(b) and 3(c), we present two MRGs of two deformed 3D faces at level 3, we concluded that MRGs are closer enough even if size is different.

\section{GRAPH MATCHING}

The matching strategy [9] of two MRGs is based on the detection of nodes with similar topological configurations in term of connections to their neighbours nodes and parents/children relations. The aim is to maximize the number of corresponding nodes. For this purpose a set of topological consistency criteria were stated. The pairs of nodes which verify the criteria are candidates for the matching. Assuming known the list of matching nodes, a function is required to measure the similarity between two $3 \mathrm{D}$ faces.

First, a function calculates the similarity between each matched nodes. The original function introduced by [9] has been enriched with mean curvature as a local geometric properties of the surface.

Assuming two 3D faces $F_{1}$ and $F_{2}$, the function used to calculate their similarity is obtained by summing the similarity, function of the matching pairs of nodes:

1. Self-identity $\operatorname{SIM}\left(F_{1} ; F_{2}\right)=1$

2. Positivity: $\operatorname{SIM}\left(F_{1} ; F_{2}\right) \geq 0$

3. Symmetry: $\operatorname{SIM}\left(F_{1} ; F_{2}\right)=\operatorname{SIM}\left(F_{2} ; F_{1}\right)$

\section{EXPERIMENTS AND RESULTS}

In this section, we present the different results obtained with the methods presented in the above sections. Firstly, our 144
3D face models and the necessary pre-processing steps are presented. Then our main algorithm is given. At last, the final tests and results are exposed.

\subsection{Dataset}

Our test dataset is made of twelve 3D faces, extracted from the database used in [4] that were digitized with Minolta 3D digitizers [14]. For each element, twelve instances have been computed using different deformations on each one. The deformations [15] have been used as a limited shapechanging transformation of the mesh: bend, twist, stretch, dent, etc. Hence, our dataset is composed of 144 3D face models categorized in 12 different classes. Most of the faces have a high resolution (40,000 triangles). Figure 2 gives an example of a digitized face.

\subsection{Pre-processing}

The main difficulty in the application of the topology-based tools is the necessity to adapt concepts developed for smooth manifold to triangulated surface, which are not necessary continous.

Due to the 3D digitizer, the scanned faces contain significant number of missing faces that forms holes in the mesh. Moreover some faces have sets of polygons that are completly disjointed from the rest of the mesh, mis-oriented, and of irregular size from the rest of the mesh. Successful practical application of $3 \mathrm{D}$ face recognition must be adapted to such surfaces. It is important to point out that 3D shape is not independent from illumination while conditions do affect the quality of sensed 3D data.

The preprocessing phase apply a holes interpolation, triangles orientation, topology refinement using the functions of Gnu Triangulated Surface tools GTS [16] (an Open Source library intended to provide a set of useful functions to deal with 3D surfaces meshed with interconnected triangles) to make our recognition system more efficient.

\subsection{Main algorithm}

The main algorithm is as follows: 1 - mesh refinement; 2 - $f$-values computation; 3 - MRG construction based on $f$ values; and 4 - MRGs comparison using the method described in [9].

\subsection{Similarity matrix}

Using Reeb graph technique, we resampled models from the datasets and performed a one-to-one matching for each possible pair of faces. Based on the results, we constructed the similarity matrix shown in figure 4 . If the algorithm is not sensitive to small difference between $Z_{\min }$ and $Z_{\max }$, we get higher similarities for pairs from the same class. 


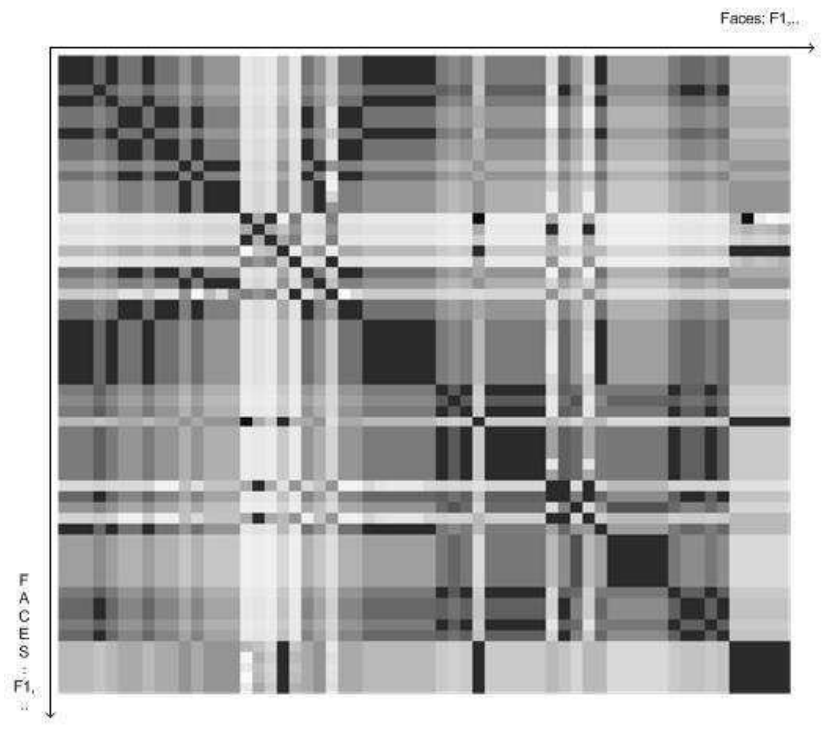

Figure 4. Similarity matrix: a dark region represents a high similarity.

The similarity matrix (figure 4) shows that the multiresolution Reeb graph technique produces accurate recognition results: we obtain rather dark regions inside the class along the diagonal, while other regions appear lighter.

To give an idea of the time needed to compute graphs, a level-3 graph requires approximatively 2 seconds of computation and a level-5 graph requires less than 6 seconds (for a face with 40,000 triangles). The comparison of two 3D faces takes less than 2 milliseconds on a $\mathrm{P} 4-3 \mathrm{GHz}$.

\section{CONCLUSIONS AND FUTURE WORKS}

We presented in this paper a topological approach for 3D face recognition, combining with geometric features by adding curvatures attributes to graph nodes.

The evaluation experiments showed that our approach gives satisfactory results in face recognition among a 144 $3 \mathrm{D}$ face models dataset. It is also important to point out that the computation time needed to extract MRG is very low, as the matching time. Consequently, our recognition system could be easily adapted to interactive time applications.

\section{ACKNOWLEDGEMENTS}

We wish to express gratitude to A. Srivastava for the use of figures from their papers and for his helpful discussions. We would like to acknowledge Laboratory for Computational Vision, Florida State University, USA, for the use of their database [4].

\section{REFERENCES}

[1] K. W. Bower, K. Chang, and P. Flynn, "A survey of approaches to three-dimensional face recognition," in ICPR, 2004, vol. 1, pp. 358-361.

[2] G. Gordon, "Face recognition based on depth maps and surface curvature," in Geometric Methods in Computer Vision, SPIE 1570, July 1991, pp. 1-12.

[3] A. B. Moreno, A. Sonchez, J. F. Velez, and F. J. Diaz, "Face recognition using 3D surface-extended descriptors," in IMVIP, September 2003.

[4] A. Srivastava, X. Liu, and C. Hesher, "Face recognition using optimal linear components of range images," Journal of Image and Vision Computing, 2003.

[5] X. Lu, D. Colbry, and A. K. Jain, "Three-dimensional model based face recognition," in ICPR, August 2004, vol. 1, pp. 362-366.

[6] J. C. Lee and E. Milios, "Matching range images of human faces," in ICCV, July 1990, pp. 722-726.

[7] V. Blanz and T. Vetter, "Face recognition based on fitting a 3D morphable model," IEEE Trans. on PAMI, vol. 25, no. 9, pp. 1063-1074, september 2003.

[8] S. Biasotti, S. Marini, and G. Patené, "An overview on properties and efficacy of topological skeletons in shape modeling," in SMI, 2003, pp. 245-254.

[9] T. Hilaga, Y. Shinagawa, T. Kohmura, and T. Kunii, "Topology matching for fully automatic similarity estimation of 3D shapes," in ACM SIGGRAPH, 2001, pp. $203-212$.

[10] A. T. Fomenko and T.L. Kunii, Topological Modeling Visualization, Springer-Verlag, 1998.

[11] G. Reeb, “Sur les points singuliers d'une forme de pfaff completement integrable," Comptes Rendus de l'Académie des Sciences de Paris, pp. 847-849, 1946.

[12] S. P. Tarasov and M. N. Vyalyi, "Construction of countour trees in 3D in $\mathrm{O}(\mathrm{n} \operatorname{logn})$ steps," in $A C M S C G$, 1998 , pp. $68-75$.

[13] Andre Graman, Topologie des surfaces, Collection Sup. Presses Universitaires de France, 1971.

[14] “http://www.minoltausa.com/vivid/products/mainen.asp," .

[15] X. Lu and A. K. Jain, "Deformations analysis for 3D face matching," to appear in IEEE WACV, 2005.

[16] “http://gts.sourceforge.net," . 